\title{
Lipid-rich carcinoma of the breast that is strongly positive for estrogen receptor: a case report and literature review
}

This article was published in the following Dove Press journal:

OncoTargets and Therapy

18 March 2016

Number of times this article has been viewed

\author{
Takaaki Oba' \\ Mayu Ono' \\ Asumi lesato' \\ Toru Hanamura' \\ Takayuki Watanabe' \\ Tokiko Ito' \\ Toshiharu Kanai' \\ Kazuma Maeno' \\ Ken-ichi Ito' \\ Ayako Tateishi \\ Akihiko Yoshizawa ${ }^{2}$ \\ Fumiyoshi Takayama ${ }^{3}$ \\ 'Division of Breast, Endocrine and \\ Respiratory Surgery, Department of \\ Surgery, Shinshu University School \\ of Medicine, Matsumoto, Nagano, \\ ${ }^{2}$ Department of Laboratory Medicine, \\ Shinshu University Hospital, ${ }^{3}$ maging \\ Center, Ichinose Neurosurgical \\ Hospital, Matsumoto, Japan
}

\begin{abstract}
Lipid-rich carcinoma (LRC) of the breast is a rare breast cancer variant that accounts for $<1 \%$ of all breast malignancies. It has been reported that LRCs are negative for estrogen receptor. Here, we report a case of LRC of the breast that was strongly positive for estrogen receptor and treated with endocrine adjuvant therapy. A 52-year-old postmenopausal female noticed a lump in her right breast by self-examination and presented to our hospital. Physical examination revealed an elastic $30 \mathrm{~mm} \times 20 \mathrm{~mm}$ hard mass in the upper medial part of her right breast. The findings obtained using ultrasonography, mammography, and contrast-enhanced magnetic resonance imaging suggested breast cancer. Core needle biopsy resulted in the diagnosis of invasive carcinoma. The patient underwent mastectomy and sentinel lymph node biopsy. Histopathologically, the tumor cells were abundant in foamy cytoplasm. Because the presence of marked cytoplasmic lipid droplets was confirmed by Sudan IV staining and electron microscopic examination of the tumor and the lipid droplets were negative for periodic acidSchiff staining, the tumor was diagnosed as an LRC. Immunohistochemically, estrogen and progesterone receptors of the tumor were strongly positive, human epidermal growth factor receptor type 2 was negative, and the ratio of Ki-67-positive cells was $\sim 30 \%$. After surgery, the patient underwent combination chemotherapy with anthracycline, cyclophosphamide, and 5-fluorouracil, followed by docetaxel. Thereafter, the pateint was treated with letrozole and has remained well for 24 months with no signs of recurrence.
\end{abstract}

Keywords: breast cancer, estrogen receptor, endocrine therapy

\section{Introduction}

Lipid-rich carcinoma (LRC) of the breast is a rare form of breast cancer that accounts for $<1 \%$ of all breast malignancies according to the World Health Organization's classification of breast tumors ${ }^{1}$ and is considered to be aggressive with a poor prognosis. ${ }^{2}$ Although LRC is thought to be negative for estrogen receptors (ERs), ${ }^{2,3}$ we recently experienced a case of breast LRC that was strongly positive for ERs. To the best of our knowledge, our case is the second report of ER-positive LRC, which was treated with endocrine therapy. Here, we report a case of LRC strongly positive for ER and review the literature. Written informed consent was obtained from the patient to use her data for publication. Approval was obtained from the ethics committee of Shinshu University, School of Medicine.

\section{Case report}

A 52-year-old postmenopausal female noticed a painless lump in her right breast on self-examination and presented to our hospital. The patient had no past or family history \footnotetext{
Division of Breast, Endocrine and Respiratory Surgery, Department of Surgery, Shinshu University School of Medicine, 3-I-I Asahi, Matsumoto, Nagano, Japan

Tel +8I 263372657

Fax +8I 263372721

Email kenito@shinshu-u.ac.jp
}

Correspondence: Ken-ichi Ito 
of serious disease and received no past hormonal therapy. Physical examination revealed a firm lump in the upper medial quadrant of the right breast. The tumor was $30 \mathrm{~mm} \times 20 \mathrm{~mm}$ in size. No nipple discharge or swelling of the axillary lymph node was observed. Blood examination results, including tumor markers, were within normal limits. Mammography showed an irregularly shaped mass with a microlobulated border. Ultrasonography showed a hypoechoic $25.8 \mathrm{~mm}$ $\times 22.9 \mathrm{~mm}$ mass with an irregular and indistinct border (Figure 1). Contrast-enhanced magnetic resonance imaging revealed an irregularly shaped mass with strong enhancement in the early phase. No intraductal spread of the tumor was detected (Figure 2). Core needle biopsy of the tumor led to a diagnosis of invasive carcinoma. ${ }^{18} \mathrm{~F}$-Fluorodeoxyglucose positron emission tomography revealed no sign of distant metastasis. The patient was diagnosed as stage IIA breast cancer and underwent mastectomy and sentinel lymph node biopsy. Histopathologically, the tumor cells were abundant in foamy cytoplasm (Figure 3A and B). Sudan IV staining (Figure 3C) and electron microscopic examination of the tumor (Figure 3D) demonstrated the presence of marked cytoplasmic lipid droplets, which were negative for periodic acid-Schiff (PAS) staining (Figure 3E). Furthermore, gross cystic disease fluid protein-15 (GCDFP-15) staining (Figure $3 \mathrm{~F})$ was negative in the cytoplasm of the tumor cells. On the other hand, cytokeratin (CK) 5/6 staining was weakly positive (Figure $3 \mathrm{G}$ ), while p53 staining was positive (Figure $3 \mathrm{H}$ ). Based on these findings, the tumor was diagnosed as LRC. Immunohistochemically, ERs and progesterone receptors (PgRs) of the tumor were strongly positive (Figure 4A and B), human epidermal growth factor receptor type 2 (HER2) was negative (Figure 4C), and the ratio of $\mathrm{Ki}$-67-positive cells was $\sim 30 \%$ (Figure $4 \mathrm{D}$ ). No lymph node metastasis was detected in the axilla. Taking into consideration the high ratio of Ki-67-positive cells and aggressiveness of LRC, the patient received standard adjuvant chemotherapy of anthracycline, cyclophosphamide, and 5-fluorouracil, followed by docetaxel. Thereafter, the patient received letrozole and has remained well for 24 months with no signs of recurrence.

\section{Discussion}

LRC is histopathologically characterized by cells with numerous optically free vacuoles of various sizes in the cytoplasm that are positive for Sudan IV staining. ${ }^{1}$ Although it is important to differentiate LRC from other vacuolated or clear cell tumors, such as glycogen-rich and apocrine carcinomas, these tumors are appropriately differentiated by special staining or immunohistochemical staining. For example, glycogen-rich and apocrine carcinomas are positive for PAS, whereas LRC is negative. With regard to GCDFP-15 staining, apocrine carcinoma is positive and LRC is negative. , $^{1,5}$ In our case, the tumor cells were negative for PAS and GCDFP-15. Furthermore, an electron microscopic examination revealed the presence of cytoplasmic lipid droplets that were positive for Sudan IV staining, which met the criteria of LRC.

The clinical characteristics of LRC are not well known because only $\sim 70$ cases have been reported in the English literature to date. However, Shi et $\mathrm{al}^{2}$ analyzed the clinicopathological data of 49 LRCs among 3,206 patients with breast cancer and reported lymph nodes metastases in 38 $(78 \%)$ and that the 2 - and 5-year survival rates of patients with LRC were $64.6 \%$ and $33.2 \%$, respectively. Thus, LRC

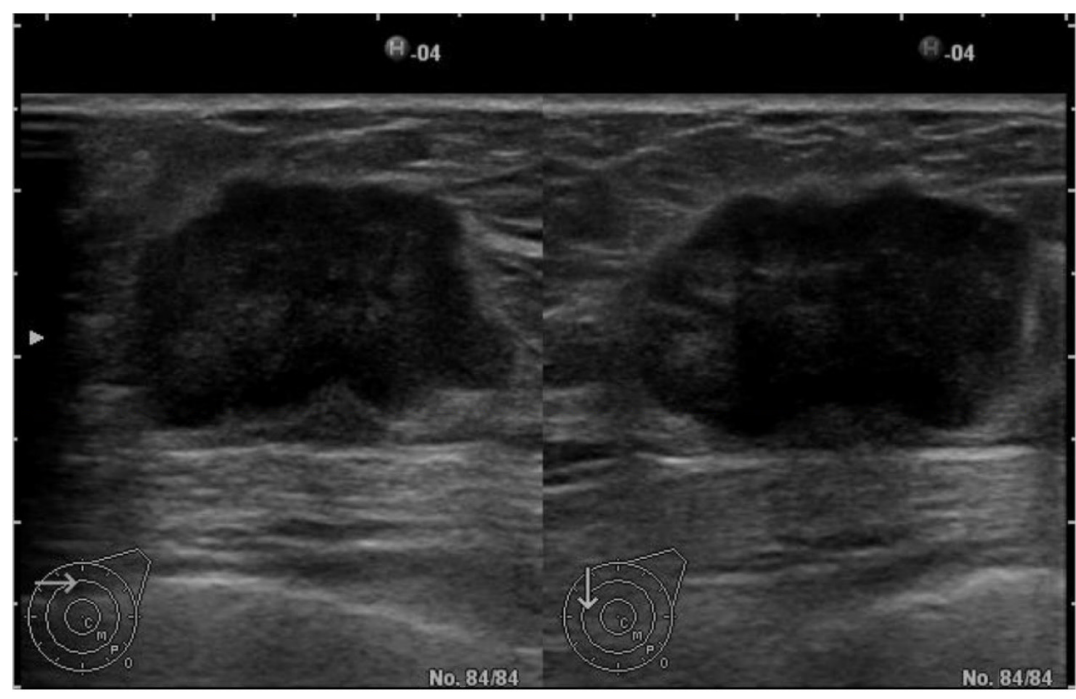

Figure I Ultrasonographic findings of the right breast.

Note: An irregularly shaped hypoechoic $25.8 \mathrm{~mm} \times 22.9 \mathrm{~mm}$ mass with an indistinct border was detected. 

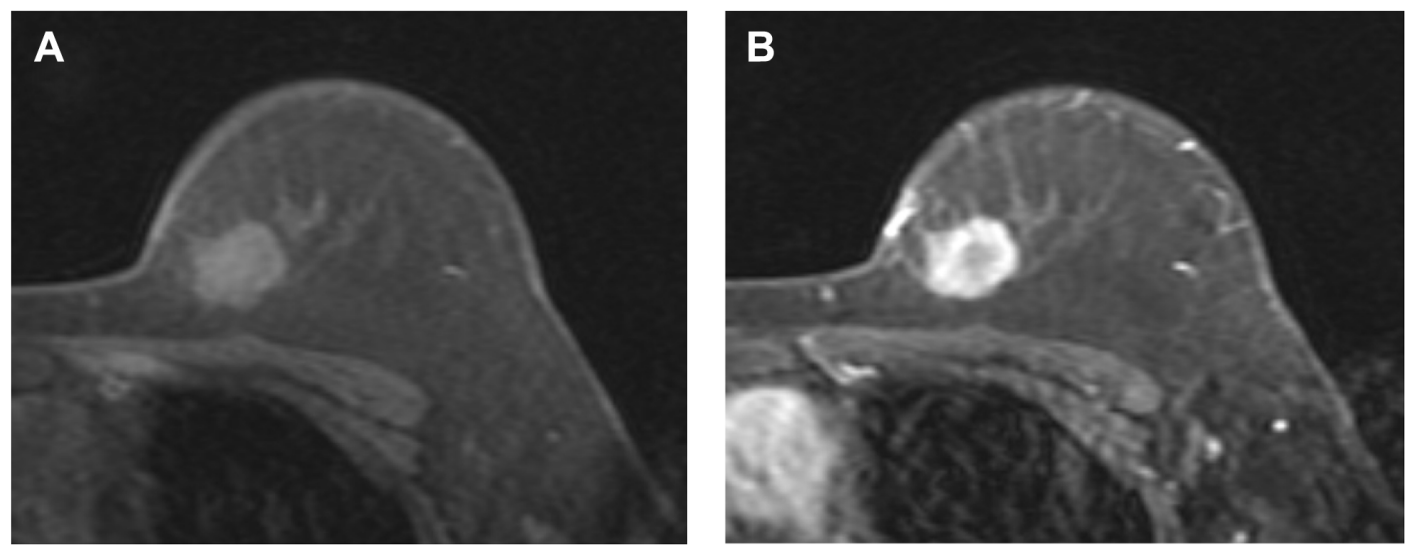

Figure 2 Contrast-enhanced magnetic resonance imaging findings of the right breast.

Notes: Contrast-enhanced magnetic resonance imaging revealed an irregularly shaped mass with strong enhancement in the early phase. (A) Before the injection of contrast agent. (B) One minute after the injection of contrast agent.

is generally considered to be an aggressive phenotype of breast cancer.

Because of the rarity of LRC, the association between the subtype and clinical features of LRC has not been extensively studied. LRC cases reported in the English literature are summarized in Table $1 .^{2,3,6,7-14}$ Guan et al ${ }^{3}$ investigated the clinicopathological features of 17 patients with LRC and reported that none were ER positive, whereas one (5.9\%) was $\mathrm{PgR}$ positive; however, all patients were HER2 positive. Shi et $\mathrm{al}^{2}$ studied 49 cases of LRC and reported that none were ER positive, five (10.2\%) were PgR positive, and 35 (71.4\%) were HER2 positive. The ratios of HER2-positive cancer in both studies were higher than the general average of $20 \%-30 \%{ }^{6,15}$ In addition, $>30 \%$ of Ki-67-positive tumor cells were detected in $27(55.1 \%)$ cases in the study by Shi et al. ${ }^{2}$ Moreover, LRC was ER negative in 12 of the
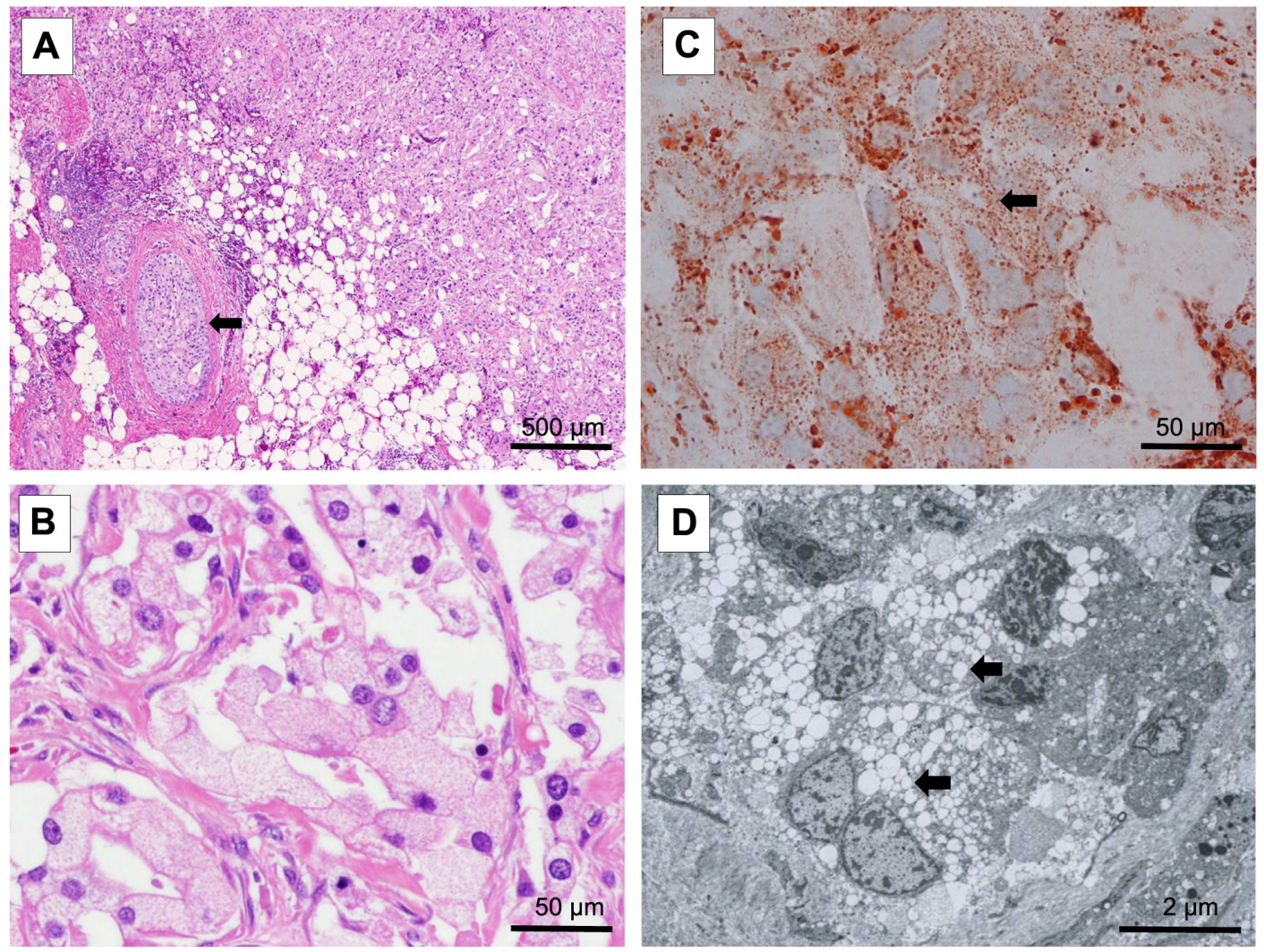

Figure 3 (Continued) 

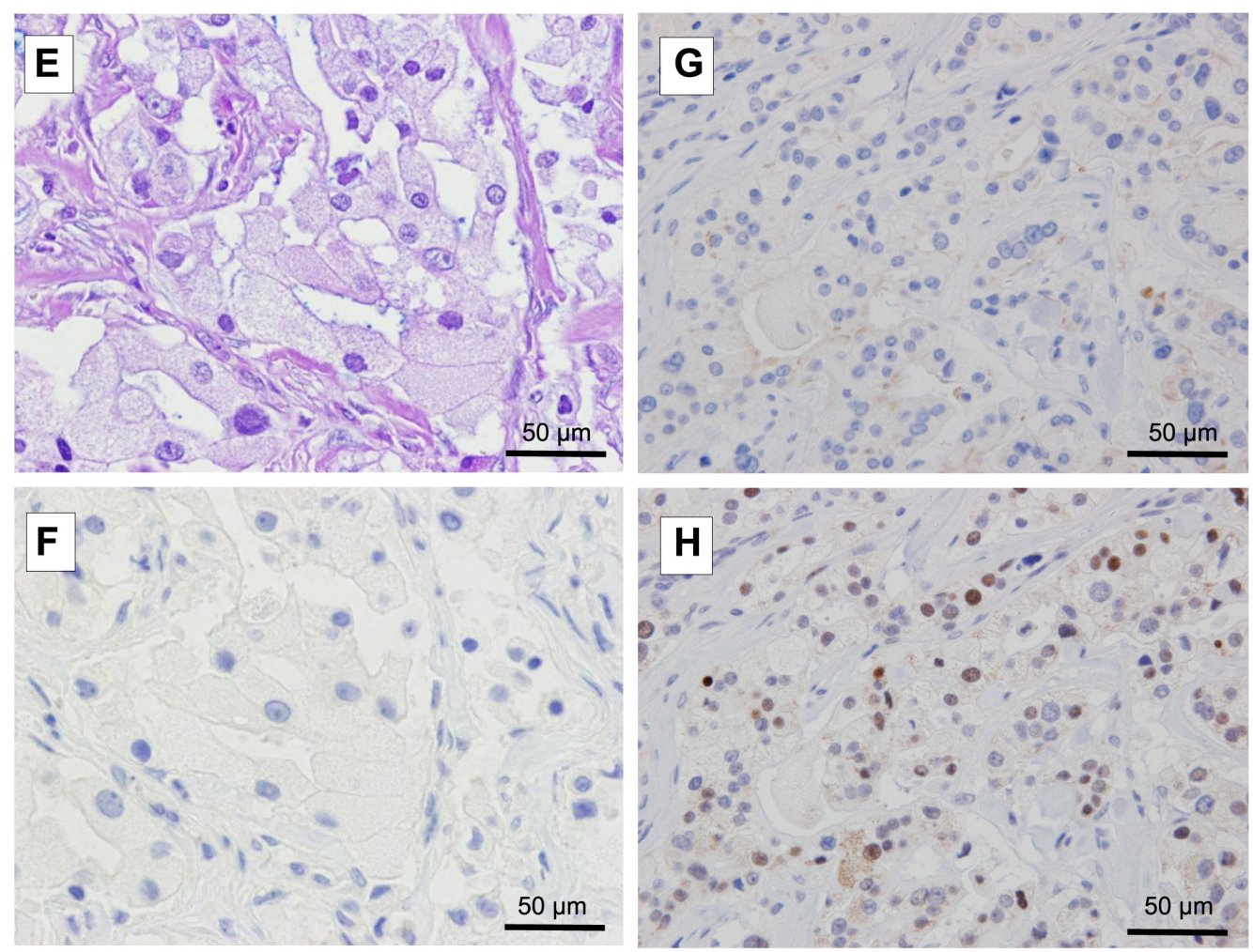

Figure 3 Histopathological findings of the lipid-rich carcinoma.

Notes: Tumor cells with abundant foamy cytoplasm and in situ neoplasm were present (arrow). Hematoxylin and eosin staining (A: $\times 40 ; \mathbf{B}: \times 400)$. Sudan IV staining $(\mathbf{C}: \times 400)$ and electron microscopic examination (D: $\times 400)$ confirmed the presence of cytoplasmic lipid droplets (arrows) that were negative for PAS staining (E: $\times 400)$. GCDFP-I5 staining (F: $\times 400)$ was negative for the cytoplasm of the tumor cells. Cytokeratin $(C K) 5 / 6(\mathbf{G}: \times 400)$ was weakly positive, and $\mathrm{p} 53$ was positive in the tumor cells $(\mathbf{H}: \times 400)$.

Abbreviations: PAS, periodic acid-Schiff; GCDFP-15, gross cystic disease fluid protein- 15.
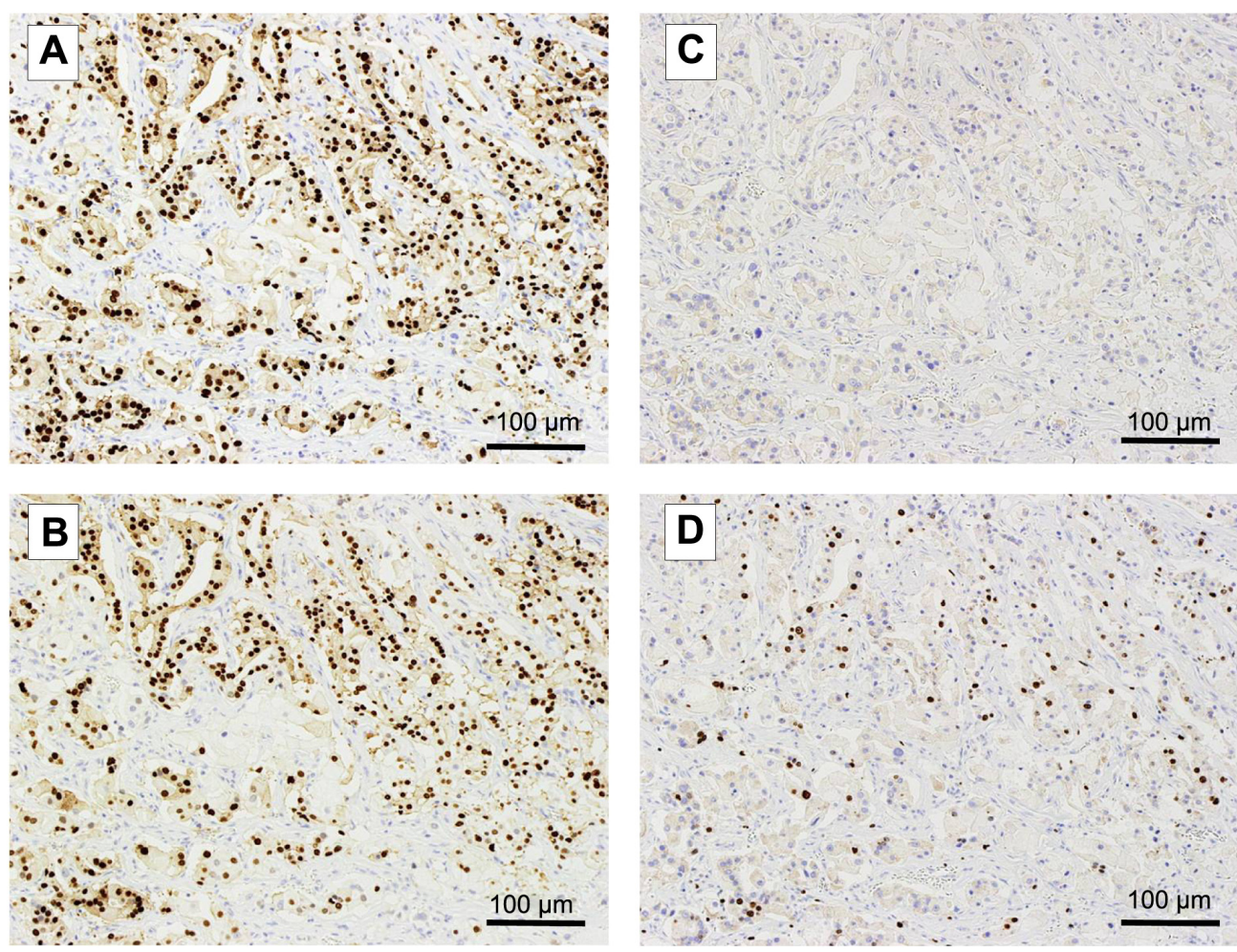

Figure 4 Immunohistochemical findings of the lipid-rich carcinoma.

Notes: More than $90 \%$ of the tumor cells were positive for estrogen receptors $(\mathbf{A}: \times 200), \sim 70 \%$ were positive for progesterone receptors $(\mathbf{B}: \times 200)$, all were negative for human epidermal growth factor receptor type $2(\mathbf{C}: \times 200)$, and the positive rate of Ki-67 was $\sim 30 \%(\mathbf{D}: \times 200)$. 


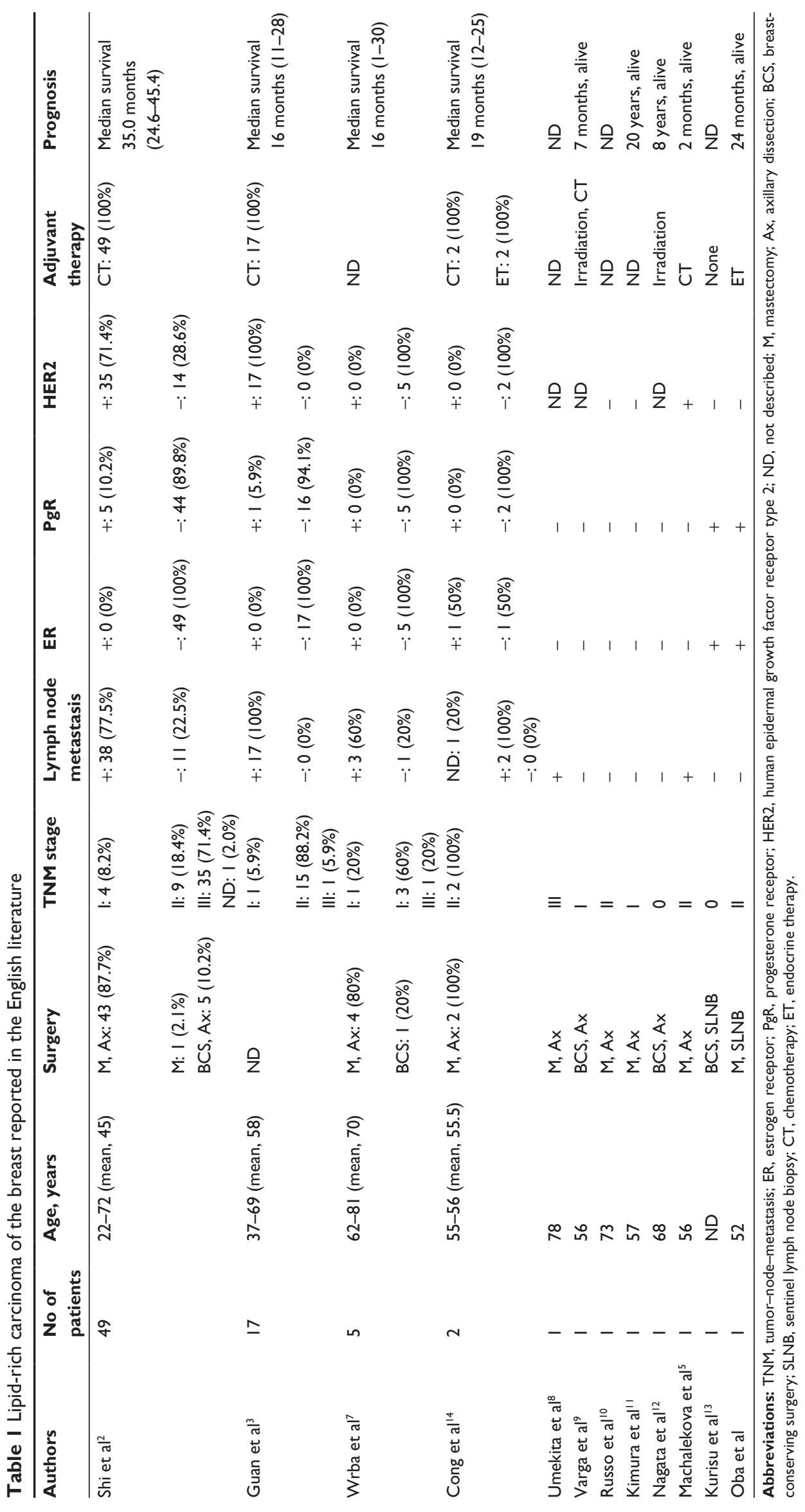


14 case reports. ${ }^{5,8-14}$ Thus, all LRCs except for two cases ${ }^{13,14}$ have been reported to be ER negative, and there has been a tendency for LRC to be HER2 positive and contain highly proliferative cells. Hence, the aggressiveness of LRC may be attributed to these biological features. However, the significance of Ki-67 levels in LRC has been not elucidated. Shi et $\mathrm{al}^{2}$ reported that $\mathrm{Ki}-67$ status was not associated with overall survival among 49 patients with LRC. However, more clinical data are required to elucidate the significance of $\mathrm{Ki}-67$ in the prognosis of LRC.

Treatment of LRC is typically performed on the basis of standard treatment protocols for breast cancer. However, considering the aggressiveness of LRC, systemic therapy should be an important part of the treatment regimen and appropriate systemic therapies according to the subtype and stage of each tumor should be administered. Shi et $\mathrm{al}^{2}$ performed an in vitro chemosensitivity assay and found that lipid-rich tumors were sensitive to paclitaxel, carboplatin, and cisplatin. Thus, chemotherapy including paclitaxel or platinum agents may have the potential to improve the prognosis of recurrent LRC. On the other hand, because all LRCs except for two previously reported cases ${ }^{13,14}$ have been ER negative, the effect of endocrine therapy on LRC has not been established. In the present case, endocrine therapy is expected to be effective because the tumor was strongly ER positive.

\section{Conclusion}

The present case is the second report of LRC that was positive for ER expression and treated with endocrine therapy. Although LRC is generally considered to be negative for ERs, our case suggests that a small percentage of LRCs are ER positive.

\section{Acknowledgment}

Written informed consent was obtained from the patient to use her data for publication.

\section{Disclosure}

The authors report no conflicts of interest in this work.

\section{References}

1. Eusebi V, Ichihara S, Vincent-Salomon A, et al. Exceptionally rare types and variants. In: Lakhani SR, Ellis IO, Schnitt SJ, editors. WHO Classification of Tumours of the Breast. 4th ed. Lyon: IARC Press; 2012:74.

2. Shi P, Wang M, Zhang Q, Sun J. Lipid-rich carcinoma of the breast. A clinicopathological study of 49 cases. Tumori. 2008;94:342-346.

3. Guan B, Wang H, Cao S, et al. Lipid-rich carcinoma of the breast clinicopathologic analysis of 17 cases. Ann Diagn Pathol. 2011;15: 225-232.

4. Satoh F, Umemura $S$, Osamura RY. Immunohistochemical analysis of GCDFP-15 and GCDFP-24 in mammary and non-mammary tissue. Breast Cancer. 2000;7:49-55.

5. Machalekova K, Kajo K, Bencat M. Unusual occurrence of rare lipidrich carcinoma and conventional invasive ductal carcinoma in the one breast: case report. Case Rep Pathol. 2012;2012:387045.

6. Kaptain S, Tan LK, Chen B. Her-2/neu and breast cancer. Diagn Mol Pathol. 2001;10:139-152.

7. Wrba F, Ellinger A, Reiner G, Spona J, Holzner JH. Ultrastructural and immunohistochemical characteristics of lipid-rich carcinoma of the breast. Virchows Arch A Pathol Anat Histopathol. 1988;413:381-385.

8. Umekita Y, Yoshida A, Sagara Y, Yoshida H. Lipid-secreting carcinoma of the breast: a case report and review of the literature. Breast Cancer. 1998;5:171-173.

9. Varga Z, Robl C, Spycher M, Burger D, Caduff R. Metaplastic lipidrich carcinoma of the breast. Pathol Int. 1998;48:912-916.

10. Russo S, Coppola D, Vinaccia P. Lipid-rich histology in a basal-type immuno-profile breast carcinoma: a clinicopathological histochemical and immunohistochemical analysis of a case. Rare Tumors. 2009;1:e41. doi:10.4081/rt.2009.e41.

11. Kimura A, Miki H, Yuri T, Hatano T, Tsubura A. A case report of lipid-rich carcinoma of the breast including histological characteristics and intrinsic subtype profile. Case Rep Oncol. 2011;4:275-280.

12. Nagata $Y$, Hanagiri T, Ono K, et al. A non-invasive form of lipidsecreting carcinoma of the breast. Breast Cancer. 2012;19:83-87.

13. Kurisu Y, Tsuji M, Shibayama Y. Intraductal lipid-rich carcinoma of the breast with a component of glycogen-rich carcinoma. J Breast Cancer. 2012;15:135-138.

14. Cong Y, Lin J, Qiao G, et al. Lipid-rich carcinoma of the breast: a report of two cases and a literature review. Oncol Lett. 2015;9:1729-1732.

15. Slamon DJ, Leyland-Jones B, Shak S, et al. Use of chemotherapy plus a monoclonal antibody against HER 2 for metastatic breast cancer that overexpresses HER2. N Engl J Med. 2001;344:783-792.
OncoTargets and Therapy

\section{Publish your work in this journal}

OncoTargets and Therapy is an international, peer-reviewed, open access journal focusing on the pathological basis of all cancers, potential targets for therapy and treatment protocols employed to improve the management of cancer patients. The journal also focuses on the impact of management programs and new therapeutic agents and protocols on

\section{Dovepress}

patient perspectives such as quality of life, adherence and satisfaction. The manuscript management system is completely online and includes a very quick and fair peer-review system, which is all easy to use. Visit http://www.dovepress.com/testimonials.php to read real quotes from published authors. 